\title{
Influence of aerobic fitness on the correspondence between heart rate variability and ventilatory threshold
}

http://dx.doi.org/10.11606/1807-5509202000040555

\begin{abstract}
The aims of this study were to verify the correspondence between heart rate variability (HRV) and ventilatory thresholds during a progressive exercise test and the relationship with low and high aerobic fitness levels. Twenty male volunteers $(29.5 \pm 6.2$ years; $75.9 \pm 13.0 \mathrm{~kg} ; 175.0 \pm 7.4 \mathrm{~cm})$ were recruited. The subjects were allocated to two groups according to their $\mathrm{VO}_{2} \max <48.8 \mathrm{ml} \bullet \mathrm{kg}^{-1} \bullet \mathrm{min}^{-1}$ (low cardiorespiratory fitness group) $(n=10)$ and $>48.81 \mathrm{ml} \bullet \mathrm{kg}^{-1} \bullet \mathrm{min}^{-1}$ (high cardiorespiratory fitness group) $(n=10)$. A progressive test was performed, consisting of 3-min stages beginning at 25 watts and increasing by 25 watts every 3 -min. The HRV threshold (HRVT) and ventilatory threshold (VT) analyses were performed through visual inspection. The comparisons with RMSSD values in percentage of maximum workload resulted in a higher effect size (ES) than the SDNN values. The $\mathrm{VO}_{2}$ in the high cardiorespiratory fitness group at VT $(+32 \%), \mathrm{HRVT}_{\text {RMSSD }}(+27 \%)$, and $\mathrm{HRVT}_{\text {SDNN }}(+31 \%)$ was significantly higher compared to the group with low cardiorespiratory fitness.

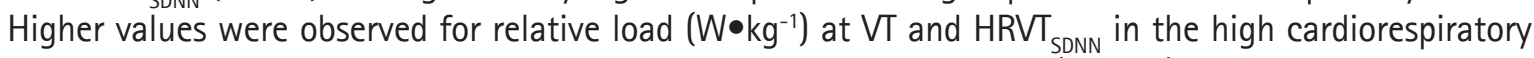
fitness group in comparison with the low cardiorespiratory fitness group $(P<0.05)$, but no difference for $V T$ and $\mathrm{HRVT}_{\text {RMSSD }}$. Significant correlations between at VT and $\mathrm{HRVT}_{\text {SDNN }}(\mathrm{r}=0.77)$ were found only in the low cardiorespiratory fitness group. Cardiorespiratory fitness should be regarded as a factor for HRVT evaluation. The HRVT $_{\text {SDNN }}$ was closer to the VT in the low cardiorespiratory fitness group than the $\mathrm{HRVT}_{\text {RMSSD'}}$, however, the use of vagal modulation assessed using the HRV parameter was more sensitive to observe possible differences regarding cardiorespiratory fitness.
\end{abstract}

KEYWORDS: Cardiac autonomic responses; Incremental test; Heart rate variability analysis; Cardiorespiratory fitness.

\section{Introduction}

The autonomic nervous system (ANS) is the primary physiological pathway which regulates heart rate (HR) by means of sympathetic and parasympathetic activities on the sinus node ${ }^{1}$. In response to exercise stress, $\mathrm{HR}$ is accelerated due to cardiac vagal withdrawal concomitant with increased sympathetic outflow ${ }^{2}$. The beat-to-beat oscillation of HR [i.e., heart rate variability (HRV)] is a non-invasive method to assess changes in the balance between the ANS branches ${ }^{3-5}$. Heart rate variability at rest and during low-intensity exercise has been shown to be positively correlated with aerobic fitness ${ }^{6}$ and responsive to aerobic and sports training effects $\mathrm{S}^{4,7,8}$.

In response to the physiological stress caused by a progressive exercise test, pulmonary ventilation increases in a non-linear fashion, as a reflection of the subtle increase in the muscular anaerobic contribution to ATP resynthesis? ${ }^{9}$ In theory, the ventilatory threshold 
(VT) corresponds to the rise in blood lactate above resting levels [i.e., lactate threshold (LT) $]^{10}$. Recently, HRV responses during incremental tests have been used to determine the heart rate variability threshold (HRVT), which is purported to coincide with VT and $\mathrm{LT}^{11}$. According to KARAPETIAN $^{11}$, HRVT can be defined as the point at which there is no further decline in time-domain HRV indices, thus indicating vagal withdrawal, however, the correspondence between HRVT, VT, and LT was tested in subjects with a wide variation in aerobic fitness. A close examination of figure 3 in of the Karapetian's ${ }^{11}$ paper reveals considerable individual discrepancies in the comparison between autonomic and metabolic thresholds. This suggests that the high correlation between HRVT and VT $(r$ $=0.89$; ranging from $-0.8-2.5 \mathrm{~L} / \mathrm{min}$ of oxygen output consideringmeans of HRVT and VT) may be weaker in a more homogeneous groups (i.e., it is possible that there is a low correlation between the VT and HRVT when analyzed separately between individuals with low and high means of HRVT and VT). It is suggested that increases in sympatheticadrenal activity and subsequent catecholamine release are responsible for stimulating glycogenolysis

\section{Methods}

The study involved two groups, divided according to aerobic fitness levels (low and high). For each group, oxygen consumption $\left(\mathrm{VO}_{2}\right)$ at VT and at HRVT were determined from same progressive cycling test. One subject from the low maximal oxygen consumption $\left(\mathrm{VO}_{2} \max \right)$ group was excluded from the analyses due to abnormal ventilatory responses which precluded determination of VT. The $\mathrm{VO}_{2}$ at VT and HRVT was compared within and between groups to ascertain the role of aerobic fitness, as assessed by $\mathrm{VO}_{2} \max$, on the cardiac autonomic response to exercise and on the correspondence between HRVT and VT. According to the median of $\left(48.8 \mathrm{ml} \bullet \mathrm{kg}^{-1} \bullet \mathrm{min}^{-1}\right)$, the participants were evenly split into two groups: low cardiorespiratory fitness group (range: $37.2-44.2 \mathrm{ml} \bullet \mathrm{kg}^{-1} \bullet \mathrm{min}^{-1} ; \mathrm{n}=10$ ) and high cardiorespiratory fitness group (range: $49.5-61.3 \mathrm{ml} \bullet \mathrm{kg}^{-1} \bullet \mathrm{min}^{-1} ; \mathrm{n}=10$ ). The cut-off value established for high cardiorespiratory fitness group was near the $95 \%$ percentile of assumed as -50 and $47.3 \mathrm{ml} \cdot \mathrm{kg}^{-1} \cdot \mathrm{min}^{-1}$ for men aged $25-34$ and $35-44^{16}$, respectively. and ventilation breakpoints ${ }^{11}$. Although it is tempting to mechanistically link HRVT, LT, and VT, it is accepted that is some cases this correspondence may fail, indicating that factors other than sympathetic outflow and anaerobic glycolysis can stimulate pulmonary ventilation ${ }^{12,13}$, such as the vagal autonomic tone resulting in fast parasympathetic withdrawal in oscillations of $\mathrm{HR}$ in individuals with higher aerobic fitness $^{14,15}$, during progressive tests. This hypothesis could indicate reduced influence of the respiratorymetabolic mechanisms linked to the occurrence of HRVT in individuals with higher aerobic fitness.

The lack of correspondence between HRVT and VT may reveal differing mechanistic bases underlying these threshold intensities and raises concerns on the use of the former as a simple and non-invasive alternative to aerobic capacity evaluation and training intensity prescription, to replace VT. Therefore, the aims of this study were threefold: 1) to compare the HRV changes during progressive exercise performed by groups possessing low and high aerobic fitness levels; 2 ) to compare the HRVT and VT between groups with low and high aerobic fitness levels and; 3 ) to verify the correspondence between HRVT and VT within each aerobic fitness group.

Twenty male volunteers aged between 20 and 44 years of age $(29.5 \pm 6.2$ years) were recruited. Prior to data collection, all participants were initially surveyed to gather information on their general characteristics. Individuals were included in the study if they met the following inclusion criteria: were healthy with physically active or sedentary lifestyles in the previous six months [using the International Physical Activity Questionnaire criteria (http://www.ipaq.ki.se/)], nonsmokers, presented no cardiovascular dysfunction (asked in an anamnesis procedures considering the last medical appointment), and were not under drug administration within at least four weeks before of the incremental test. All participants signed an informed consent and the procedures were approved by the local Ethics Committee (process number 091/2013).

TABLE 1 displays the physical and physiological characteristics of the low and high cardiorespiratory fitness groups. There were no significant differences between groups regarding age, height, and HRmax. The cardiorespiratory fitness (and maximal power output 
- Wmax) was higher for the group with high aerobic fitness in comparison with the group with low aerobic fitness. Body weight and BMI were also higher in the lower aerobic fitness group.

TABLE 1 - Physical and physiological characteristics of the low cardiorespiratory fitness group; $(n=10)$ and high cardiorespiratory fitness group; $(\mathrm{n}=10)$ from the median of $\mathrm{VO}_{2} \max \left(48.7 \mathrm{ml} \bullet \mathrm{kg}^{-1} \bullet \mathrm{min}^{-1}\right)$. Mean values, ( \pm standard deviation) and confidence intervals [CI 95\%].

\begin{tabular}{|c|c|c|c|}
\hline & $\begin{array}{l}\text { Low cardiorespiratory } \\
\text { fitness group }\end{array}$ & $\begin{array}{l}\text { High cardiorespiratory } \\
\text { fitness group }\end{array}$ & $P$ \\
\hline \multirow{2}{*}{ Age (years) } & $30.3 \pm 6.1$ & $28.6 \pm 6.4$ & 0.55 \\
\hline & {$[25.9-34.6]$} & {$[23.9-33.2]$} & \\
\hline \multirow{2}{*}{ Weight (kg) } & $83.9 \pm 13.8$ & $67.8 \pm 4.7$ & $<0.02^{*}$ \\
\hline & {$[74.0-93.8]$} & {$[64.4-71.2]$} & \\
\hline \multirow{2}{*}{ Height $(\mathrm{cm})$} & $174.9 \pm 8.4$ & $175.8 \pm 6.4$ & 0.61 \\
\hline & {$[168.0-180.2]$} & {$[171.3-180.4]$} & \\
\hline \multirow{2}{*}{ BMI $\left(\mathrm{kg} \bullet \mathrm{m}^{-2}\right)$} & $27.7 \pm 3.9$ & $22.0 \pm 2.0$ & $<0.01^{*}$ \\
\hline & {$\left[\begin{array}{ll}24.9 & -30.4\end{array}\right]$} & {$[20.5-23.4]$} & \\
\hline \multirow{2}{*}{$V O_{2} \max \left(\mathrm{ml} \bullet \mathrm{kg}^{-1} \bullet \mathrm{min}^{-1}\right)$} & $41.3 \pm 3.5$ & $56.2 \pm 4.1$ & $<0.01^{*}$ \\
\hline & {$[38.8-43.8]$} & {$[53.3-59.1]$} & \\
\hline \multirow{2}{*}{$V O_{2} \max \left(\mathrm{L} \cdot \mathrm{min}^{-1}\right)$} & $3.4 \pm 0.4$ & $3.8 \pm 0.3$ & $<0.05^{*}$ \\
\hline & {$[3.1-3.7]$} & {$[3.6-4.1]$} & \\
\hline \multirow{2}{*}{ HRmax (bpm) } & $189.1 \pm 11.2$ & $188.8 \pm 10.9$ & 0.95 \\
\hline & {$[181.1-197.1]$} & {$[181.0-196.5]$} & \\
\hline \multirow{2}{*}{ Wmax (W) } & $267.2 \pm 28.6$ & $300.0 \pm 29.8$ & $<0.03^{*}$ \\
\hline & {$[246.8-287.7]$} & {$[278.7-321.3]$} & \\
\hline
\end{tabular}

* Significant difference using independent student T-test; $\mathrm{BMI}=$ body mass index;

$\mathrm{VO}_{2}=$ maximum oxygen output; HRmax = maximal heart rate; Wmax = maximal power from incremental test.

\section{Procedures}

\section{Exercise testing}

Subjects were instructed to avoid food or beverages containing caffeine for 24 hours and vigorous exercise for 48 hours prior to testing. Upon arrival at the laboratory, body mass and height were measured with a calibrated scale and a stadiometer to the nearest 0.02 $\mathrm{kg}$ and $0.1 \mathrm{~cm}$, respectively. The body mass index (BMI) was calculated by dividing the weight $(\mathrm{kg})$ by the square of the height $(\mathrm{cm})$.

The progressive test was performed on an electromagnetically braked cycle ergometer (LODE, Excalibur Sport, Groningen, Netherlands) in a laboratory with a controlled temperature $\left(-23^{\circ} \mathrm{C}\right)$, and a fan directed to the participant when required, in order to improve thermal comfort during the test. The testing protocol consisted of 3 -min stages ${ }^{11}$ beginning at 25 watts and increasing 25 watts every $3-\min ^{15}$. 
The 3-min stages were chosen for the progressive test as this enables a higher quality stationary signal of $\mathrm{RR}$ intervals to be obtained. Subjects were instructed to maintain a pedal cadence of between 60 and 90 revolutions per $\min (\mathrm{rpm})$. The exercise test duration ranged from 15 to $40 \mathrm{~min}$, depending on each subject's cardiorespiratory fitness. Subjects finished the test when they reached volitional exhaustion or felt unable to maintain the pedal cadence $>60 \mathrm{rpm}$. Throughout the test, participants were verbally motivated to give their best performance.

\section{Determination of heart rate variability threshold (HRVT)}

Heart rate variability was measured using a telemetric device (Polar RS800 Electro Oy, Kempele, Finland) used to record each subject's RR intervals (beat-tobeat fluctuation in HR) throughout the test. The RR interval data were stored in the receiving watch and subsequently uploaded to a computer for analysis using Polar Pro Trainer software (version 5.0). All analyses were performed using Kubios HRV Analysis Software 2.0 (Biosignal Laboratory, University of Kuopio, Finland). The RR intervals from the final $2 \mathrm{~min}$ of each progressive test stage were used for analysis of $H R V^{11}$, avoiding the influence of non-stationary RR intervals on HR oscillations, commonly observed in the early stages. The RR interval was automatically interpolated in cases where it deviated from the previous interval by $>30 \%$, followed by visual inspection to correct for noise ${ }^{15}$. The standard deviation of all RR intervals (SDNN), which represents both sympathetic and parasympathetic activity and the square root of the mean squared differences of successive RR intervals (RMSSD) in milliseconds (ms), as a parasympathetic activity indicator, were retained for analysis ${ }^{1}$. To determine the HRVT, the RMSSD and SDNN for each stage were plotted against power output (W) or percentage of maximum workload (\%W). The exercise intensity at which no further decline in SDNN and RMSSD values is observed defines the occurrence of

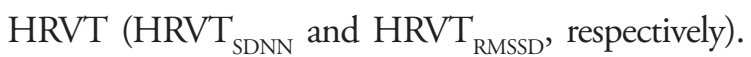
Two experienced researchers (with at least 2 years of practical experience in assessments of cardiopulmonary parameters in exercise tests) with no access to the identity of the subjects performed the HRVT analyses. In the case of disagreement between power outputs, a third independent researcher performed the HRV determination. In all cases, the third result coincided with one of the previous results and this intensity was thus defined as the individual's HRVT, or the median those three outcomes. High inter-rater intraclass correlation coefficient $(0.80)$ between the researchers considering HRVT was found.

\section{Determination of ventilatory threshold (VT) and $\mathrm{VO}_{2}$ max}

Respiratory gas exchange was measured continuously breath-by-breath using a metabolic cart (Quark CPET, Cosmed, Italy) and averaged each 20-s in order to obtain VT and $\mathrm{VO}_{2} \max$. Prior to each test, the equipment was calibrated using ambient air and gases of known $\mathrm{O}_{2}(16 \%)$ and $\mathrm{CO}_{2}(5 \%)$ concentrations. The turbine flow-meter was calibrated using a 3-L syringe. The calibration procedures followed the manufacturer's instructions.

The individual $\mathrm{VO}_{2}$ max was determined as the highest value achieved in 20-s averages close to the end of the test. Although no secondary criterion was used to validate $\mathrm{VO}_{2} \max ^{17}$, all subjects reported $>19$ rating of perceived exertion on the 6-20 Borg scale, determined in the final 30 seconds of each stage ${ }^{18}$. The VT was determined at the power output corresponding to the increase in $\mathrm{VE} / \mathrm{VO}$, without a concomitant increase in $\mathrm{VE} / \mathrm{VCO}_{2}{ }^{16}$. The power output and $\mathrm{VO}_{2}$ at $\mathrm{VT}$ have previously been considered a valid measure with high intraclass correlations using tests with increments of $25 \mathrm{~W}$ per stage ${ }^{19}$. Each individual's VT was assessed by two experienced researchers involved in the study, who were blinded to the participants' identity and group allocation $^{20}$. In cases of disagreement between the two researchers, a third researcher arbitrated. The $\mathrm{HR}, \mathrm{VO}_{2}$, and RPE at VT were retained for analyses. The heart rate (HR) and $\mathrm{VO}_{2}$ at $\mathrm{VT}$ and HRVT were calculated as the mean values from the final 2-min of the stage.

\section{Statistical analysis}

The Shapiro-Wilk's test indicated normal distribution of data. Thus, the independent Student's t test was applied for comparisons between groups (low versus high cardiorespiratory fitness group) for age, anthropometric variables (weight, height, and $\mathrm{BMI}$ ), physiological responses from progressive testing ( $\mathrm{VO}_{2}$ max, HRmax, and Wmax), physiological variables at VT, HRVT RMSSD , and $\mathrm{HRVT}_{\text {SDNN }}\left(\mathrm{VO}_{2}\right.$, $\% \mathrm{VO}_{2} \max , \mathrm{HR}, \% \mathrm{HR}$ max, RPE), load (watts), and relative load (watts ${ }^{\circ \mathrm{kg}^{-1}}$ ). One way ANOVA was used to compare the physiological and perceptual responses

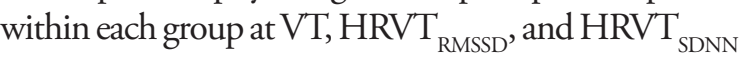
( $\left.\mathrm{VO}_{2}, \% \mathrm{VO}_{2} \max , \mathrm{HR}, \% \mathrm{HRmax}, \mathrm{RPE}\right)$, load (watts), and relative load (watts $\mathrm{kg}^{-1}$ ). The two-way repeated measures ANOVA with Bonferroni correction was used to compare the values of RMSSD and SDNN (ms) at 
the percentages of maximum workload corresponding to from $10 \%$ to $100 \%$ between the high and low cardiorespiratory fitness groups. The sphericity was checked using Mauchly's test and whenever the test was violated the necessary technical corrections were performed with the Greenhouse-Geisser test. Data are presented as mean \pm standard deviation (SD) and the significance level was set at 5\% $(P<0.05)$. Pearson's correlation was performed to assess the relationship between $\mathrm{VO}_{2}\left(\mathrm{ml} \bullet \mathrm{kg}^{-1} \bullet \mathrm{min}^{-1}\right)$ values at ventilatory

\section{Results}

The HRV (RMSSD and SDNN parameters) responses to the progressive test between the groups with different cardiorespiratory fitness levels are displayed in FIGURE 1. The pairwise comparisons between low and high cardiorespiratory fitness showed there were no main effect significant differences for RMSSD and SDNN at the percentage of maximum workload corresponding to from $10 \%$ to $100 \%$ of $\mathrm{VO}_{2}$ max. Two-way repeated measures ANOVA demonstrated main effects only when considering the percentage of maximum workload factor for RMSSD $(\mathrm{F}=27.3 ; P<0.01)$ and SDNN $(\mathrm{F}=54.4 ; P<0.01)$. The group and interaction effects between the percentage of maximum workload and group were not significant for either RMSSD $(\mathrm{F}=0.81 ; P>0.05)$ or SDNN $(\mathrm{F}=0.02 ; P>0.05)$. FIGURE 1 illustrates the HRVT identification of two typical subjects from the low (Panel C) and high (Panel D) cardiorespiratory fitness groups. In the low cardiorespiratory fitness group, it was easier to visually determine the HRVT $\mathrm{RMSSD}_{\text {D }}$ and HRVT $_{\text {SDNN }}$, and in this case they were coincident. On the other hand, in the high cardiorespiratory fitness group, it was more difficult to clearly determine the HRVT thresholds, and the HRVT ${ }_{\text {RMSSD }}$ and HRVT $_{\text {SDNN }}$ did not coincide in most cases.

TABLE 2 presents the comparisons between $\mathrm{VO}_{2}$ at $\mathrm{VT}$ and at the heart rate variability thresholds $\left(\mathrm{HRVT}_{\mathrm{RMSSD}}\right.$ and $\left.\mathrm{HRVT}_{\mathrm{SDNN}}\right)$. The $\mathrm{VO}_{2}$ at VT $(+32 \%)$,

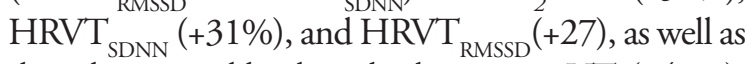
the relative workloads to body mass at VT $(+41 \%)$, HRVT $_{\text {SDNN }}(+33 \%)$, and HRVT RMSSD $(+39)$, were higher in the group with high cardiorespiratory fitness compared to the group with low cardiorespiratory fitness. There were no significant differences between the low and high cardiorespiratory fitness groups for the percentage of $\mathrm{VO}_{2} \max \left(\% \mathrm{VO}_{2} \max \right)$ at VT $(67.2 \pm 5.7$ vs $63.8 \pm 10.3 ; \mathrm{P}=0.12), \mathrm{HRVT}_{\text {RMSSD }}(61.5 \pm 8.7$ vs
(VT) and heart rate variability thresholds (HRVT $\mathrm{RMSSD}_{\text {D }}$ and HRVT $\left._{\text {SDNN }}\right)$. The aforementioned analyzes were performed in SPSS version 20.0 software for Windows. Additionally, Cohen's effect size (ES) was used to interpret the magnitude of difference between the high and low $\mathrm{VO}_{2}$ max groups considering the RMSSD and SDNN values at each percentage (from 10 to $100 \%$ of maximum workload). The threshold values for Cohen's ES statistics were $0.20-0.50$ (small), $0.50-0.80$ (moderate), and $>0.80$ (large) $)^{21}$.

56.7 $\pm 8.4 ; \mathrm{P}=0.73)$, or $\mathrm{HRVT}_{\mathrm{SDNN}}(70.0 \pm 9.8$ vs $66.1 \pm 9.4 ; \mathrm{P}=0.99)$, respectively.

The power output at VT $(144.4 \pm 20.8 \mathrm{~W}) \mathrm{did}$ not differ from the power output at $\mathrm{HRVT}_{\mathrm{RMSSD}}$ $(125.0 \pm 25.0 \mathrm{~W} ; P=0.06)$ or $\mathrm{HRVT}_{\text {SDNN }}(152.8 \pm 29.2$ $\mathrm{W} ; P=0.35)$ within the low cardiorespiratory fitness group. However, a significant difference was found between power output at HRVT $\mathrm{RMSSD}_{\text {and }}$ HRVT $_{\text {SDNN }}(P<0.01)$ in this group. Similarly, in the high cardiorespiratory fitness group the power output at VT (165.0 42.8$)$ was not different from the power output at $\mathrm{HRVT}_{\mathrm{RMSSD}}(135.0 \pm 29.3 \mathrm{~W} ; P=0.07)$ or HRVT $_{\text {SDNN }}(170.0 \pm 34.9 \mathrm{~W} ; P=0.05)$. The power output was different $(P<0.01)$ between HRVT $\mathrm{RMSSD}_{\mathrm{R}}$ and HRVT ${ }_{\text {SDNN }}$.

Heart rate expressed both in absolute values (bpm) and as a percentage of maximal heart rate (\%HRmax) was significantly different $(P<0.05)$ in the low cardiorespiratory group between the VT $(80.8 \pm 4.2$ $\%$ HRmax; $153.0 \pm 13.5 \mathrm{bpm})$ and the HRVT ${ }_{\text {RMSSD }}$ $(77.7 \pm 5.4 \% H R m a x ; 146.8 \pm 9.8 \mathrm{bpm})$, and between the VT and the HRVT ${ }_{\text {SDNN }}(84.1 \pm 4.7 \%$ HRmax; $159.2 \pm 12.3 \mathrm{bpm})$. In the high cardiorespiratory fitness group, there were no differences $(P>0.05)$ in heart rate between the VT $(78.2 \pm 7.2 \% \mathrm{HRmax}$; $147.8 \pm 17.7 \mathrm{bpm})$ and HRVT RMSSD $(77.7 \pm 5.4$ $\% H R m a x ; 139.9 \pm 10.0 \mathrm{bpm})$, or between the VT and HRVT $_{\text {SDNN }}(80.3 \pm 6.9 \% H R \max ; 151.1 \pm 11.4 \mathrm{bpm})$.

Differences were found in $\mathrm{HRVT}_{\mathrm{RMSSD}}$ and HRVT $_{\text {SDNN }}$ in both groups for $\% V O_{2}(P<0.01)$. No differences were detected between the low and high cardiorespiratory fitness groups regarding power output at VT $(P=0.21), \mathrm{HRVT}_{\text {RMSSD }}(P=0.44)$, or HRVT ${ }_{\text {SDNN }}$ $(P=0.26)$. No differences in \%HRmax at VT, HRVT $_{\text {RMSSD }}$, or HRVT $T_{\text {SDNN }}$ were identified between groups $(P=0.36,0.21,0.18$, respectively). The RPE at $\mathrm{HRVT}_{\text {RMSSD }}$ and HRVT $\mathrm{SDNN}$ were significantly 
different $(P<0.01)$ in both groups.

In addition to these findings, the rating of perceived exertion (RPE) was not different between the groups with low and high cardiorespiratory fitness at VT (12.8 \pm 1.2 vs $13.9 \pm 3.1), \mathrm{HRVT}_{\mathrm{RMSSD}}(11.8 \pm 2.2$ vs $12.7 \pm 3.4)$, or HRVT ${ }_{\text {RMSSD }}(13.2 \pm 2.5$ vs $14.8 \pm 2.5)$ ( $P>0.05)$, respectively.

Considering the HRV analyzes assuming the percentage of maximum workload from the progressive test, the Cohen's ES of the pairwise comparison was large at 70\% (ES: 0.94) and 80\% (ES: 1.10), and moderate at 60\% (ES: 0.52 ) and $100 \%$ (ES: 0.68) for RMSSD. Regarding the SDNN values, the ES was small from 10 to $100 \%$ of maximum workload. Significant differences $(P<0.05)$ and a large Cohen's ES were observed when comparing the low and high cardiorespiratory fitness for relative workloads with VT (ES: 1.50), HRVT ${ }_{\text {RMSSD }}$ (ES: 1.22), and HRVT $_{\text {SDNN }}$ (ES: 1.32). The Cohen's ES between VT and HRVT $\mathrm{RMSSD}$ within groups was 0.69 (moderate) and 0.81 (large) for the low and high cardiorespiratory fitness groups, respectively (see TABLE 2 ). When the data from both groups were pooled and power output was expressed relative to body mass (W• $\mathrm{kg}^{-1}$ ), the ES in the comparison between VT $(2.09 \pm 0.58)$ and HRVT $_{\text {RMSSD }}(1.76 \pm 0.49)$ was (ES: 0.61) and between HRVT $_{\text {RMSSD }}$ and HRVT ${ }_{\text {SDNN }}(2.20 \pm 0.61)$ (ES: 0.74) was moderate $(P<0.05)$. No difference and a small ES were observed between VT and HRVT ${ }_{\text {SDNN }}$ (ES: 0.18; P 0.05).

TABLE 3 displays the Pearson's correlation coefficients(r) between $V_{2}$ values at VT, HRVT ${ }_{\text {RMSSD' }}$ and $\mathrm{HRVT}_{\mathrm{SDNN}}$. The correlations were higher in the low cardiorespiratory fitness group than in the high cardiorespiratory fitness group. $\mathrm{VO}_{2}$ at $\mathrm{VT}(0.84$, $P<0.05$ and $0.62, P=0.06), \mathrm{HRVT}_{\mathrm{RMSSD}}(0.61, P=0.07$ and $0.48, P=0.15)$, and HRVT ${ }_{\text {SDNN }}(0.76, P<0.05$ and $0.67, P<0.05)$ demonstrated higher correlations with in the low cardiorespiratory fitness group than the high cardiorespiratory fitness group, respectively.

FIGURE 1 - Changes in mean and standard deviation of RMSSD (Panel A) and SDNN (Panel B) in percentage of maximum workload of individuals from groups with low (closed black circles) and high (gray triangle) cardiorespiratory fitness levels. HRV vs. workload of an individual from the low aerobic fitness group (Panel C) and an individual from the high aerobic fitness group (Panel D).

Panel A

$@^{\circledR}$ Significant

difference in pairwise comparisons between low and high cardiorespiratory fitness using Bonferroni's post-hoc test and large effect size $(E S>0.80)$.

\&Difference considering moderate effect size (0.500.80 ).

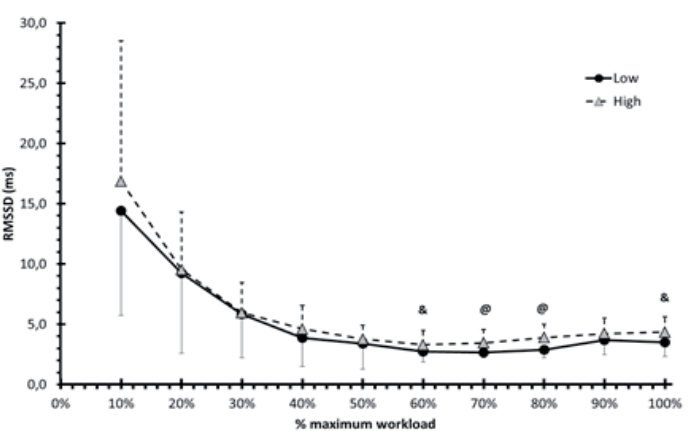

Panel C

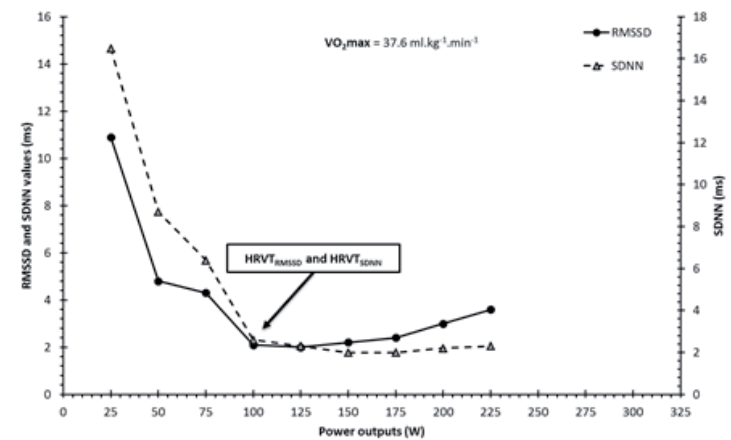

Panel B

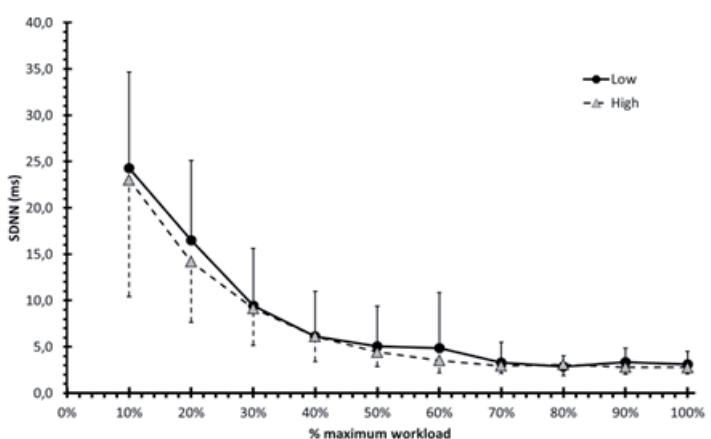

Panel D

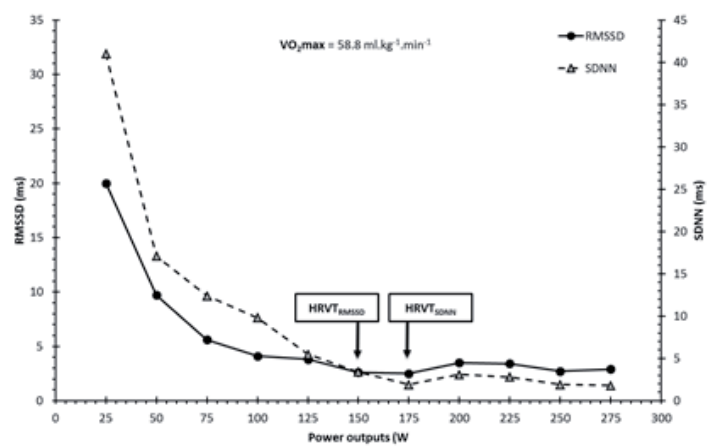


TABLE 2 - Comparisons between low $(n=9)$ and high $(n=10)$ cardiorespiratory fitness groups for $\mathrm{VO}_{2}$ $\left(\mathrm{ml} \cdot \mathrm{kg}^{-1} \bullet \mathrm{min}^{-1}\right)$, relative workload $\left(\mathrm{W} \cdot \mathrm{kg}^{-1}\right)$ and percentage of Wmax $(\%)$ corresponding to ventilatory (VT) and heart rate variability thresholds $\left(\mathrm{HRVT}_{\mathrm{RMSSD}}\right.$ and $\left.\mathrm{HRVT}_{\mathrm{SDNN}}\right)$.

\begin{tabular}{|c|c|c|c|c|c|}
\hline \multirow[b]{2}{*}{$V O_{2}\left(\mathbf{m l} \cdot \mathbf{k g}^{-1} \bullet \mathrm{min}^{-1}\right)$} & \multicolumn{2}{|c|}{ Low cardiorespiratory fitness group } & \multicolumn{2}{|c|}{ High cardiorespiratory fitness group } & \multirow{2}{*}{$\begin{array}{l}\text { Effect Size (ES): Low - High } \\
\text { cardiorespiratory fitness } \\
\text { groups }\end{array}$} \\
\hline & & & & & \\
\hline VT & 27.3 & $\pm 3.7[24.5-30.2]$ & 36.3 & $\pm 6.9^{*}[31.3-41.3]$ & 1.58 (Large) \\
\hline HRVT $_{\text {RMSSD }}$ & 25.0 & $\pm 4.3[21.7-28.4]$ & 31.9 & $\pm 5.4^{*}[28.0-35.8]$ & 1.39 (Large) \\
\hline $\mathrm{HRVT}_{\mathrm{SDNN}}$ & 28.5 & $\pm 5.1 \#[24.6-32.5]$ & 37.3 & $\pm 6.2^{*} \#[32.8-41.7]$ & 1.51 (Large) \\
\hline$E S: V T-H_{R V T} T_{\text {RMSSD }}$ & & 0.58 (Moderate) & & .71 (Moderate) & \\
\hline ES: VT - HRVT $\mathrm{SDNN}_{\mathrm{S}}$ & & 0.26 (Small) & & 0.14 (Trivial) & \\
\hline ES: $\mathrm{HRVT}_{\mathrm{RMSSD}}-\mathrm{HRVT}_{\mathrm{SDNN}}$ & & 0.74 (Moderate) & & 0.92 (Large) & \\
\hline \multicolumn{6}{|c|}{ Relative to body mass workload $\left(\mathrm{W} \bullet \mathrm{kg}^{-1}\right)$} \\
\hline VT & 1.7 & $\pm 0.3[1.5-2.0]$ & 2.4 & $\pm 0.6^{*}[2.0-2.8]$ & 1.49 (Large) \\
\hline $\mathrm{HRVT}_{\text {RMSSD }}$ & 1.5 & $\pm 0.3[1.2-1.8]$ & 2.0 & $\pm 0.5^{*}[1.7-2.3]$ & 1.22 (Large) \\
\hline $\mathrm{HRVT}_{\text {SDNN }}$ & 1.8 & $\pm 0.5[1.5-2.2]$ & 2.5 & $\pm 0.6^{*}[2.1-2.9]$ & 1.32 (Large) \\
\hline$E S: V T-H_{R V T} T_{\text {RMSSD }}$ & & 0.69 (Moderate) & & 0.81 (Large) & \\
\hline$E S: V T-H R V T_{\text {SDNN }}$ & & 0.29 (Small) & & 0.18 (Trivial) & \\
\hline$E S: \mathrm{HRVT}_{\mathrm{RMSSD}}-\mathrm{HRVT}_{\mathrm{SDNN}}$ & & 0.85 (Large) & & 1.01 (Large) & \\
\hline
\end{tabular}

* Significant different between low vs. high cardiorespiratory fitness group ( $P<0.05) ;$

\# Significant difference within low or high cardiorespiratory fitness group in comparison between HRVT $_{\text {RMSSD }}$ and HRVT $_{\text {SDNN: }}$.

TABLE 3 - Pearson product-moment correlation coefficients between $\mathrm{VO}_{2}\left(\mathrm{ml} \cdot \mathrm{kg}^{-1} \cdot \mathrm{min}^{-1}\right)$ values at ventilatory (VT) and heart rate variability thresholds (HRVT ${ }_{\mathrm{RMSSD}}$ and $\left.\mathrm{HRVT}_{\mathrm{SDNN}}\right)$.

\begin{tabular}{|c|c|c|c|c|c|c|}
\hline & \multicolumn{3}{|c|}{ Low cardiorespiratory fitness group $(\mathrm{n}=9)$} & \multicolumn{3}{|c|}{ High cardiorespiratory fitness group $(n=10)$} \\
\hline & $\mathrm{VO}_{2}$ at $\mathrm{VT}$ & $\mathrm{VO}_{2}$ at $\mathrm{HRVT}_{\mathrm{RMSSD}}$ & $\mathrm{VO}_{2}$ at $\mathrm{HRVT}_{\mathrm{SDNN}}$ & $\mathrm{VO}_{2}$ at $\mathrm{VT}$ & $\mathrm{VO}_{2}$ at $\mathrm{HRVT}_{\mathrm{RMSSD}}$ & $\mathrm{VO}_{2}$ at $\mathrm{HRVT}_{\mathrm{SDNN}}$ \\
\hline$\underset{\mathrm{MSSD}}{\mathrm{VO}_{2} \text { at } \mathrm{HRVT}_{\mathrm{R}-}}$ & 0.52 & ----- & $0.85^{*}$ & 0.38 & --.-- & $0.80^{*}$ \\
\hline $\mathrm{VO}_{2}$ at $\mathrm{HRVT}_{\text {SDNN }}$ & $0.77^{*}$ & $0.85^{*}$ & ---- & 0.39 & $0.80^{*}$ & ---- \\
\hline
\end{tabular}

${ }^{*} P<0.05$. 


\section{Discussion}

The main result of the study was that the differences between VT, HRVT $T_{\text {RMSSD }}$, and HRVT $T_{\text {SDNN }}$ were greater in the high cardiorespiratory fitness group than in the low cardiorespiratory fitness group. Thus, this finding indicates that the use of the threshold as an aerobic capacity assessment tool (first threshold) should be viewed with some caution, especially in individuals with high cardiorespiratory fitness. The rationale between VT and RR oscillations measured through vagal HRV indices is evidenced as being synchronous with respiratory sinus arrhythmia, mediated by the vagus nerve ${ }^{22,23}$. However, the greatest differences were observed in individuals who presented higher $\mathrm{VO}_{2}$ max values, although this was not noted by other authors ${ }^{11,23}$. We consider that there is a certain mismatch in physiological responses in HRV and respiratory/ventilatory dynamics in individuals with high aerobic fitness during a progressive test.

Abnormal behavior concerning ventilatory responses in progressive tests have been reported with VT determination ${ }^{24}$, as well as in one subject in the present study from the low cardiorespiratory fitness group. This fact demonstrates that VT should be analyzed carefully, requiring trained staff and gas exchange analysis equipment, which generates high cost. On the other hand, the HRVT using the visual inspection technique is an easy tool and less costly than VT, besides presenting high reproducibility ${ }^{25}$. Although the correspondence between metabolic transition thresholds and HRVT has been documented previously ${ }^{11,26}$, we found that it may present some disagreement in well-conditioned individuals who demonstrate higher rates of $\mathrm{VO}_{2} \max$, possibly due to the more pronounced physiological mechanisms underlying vagal autonomic activity being more pronounced in well-trained individuals ${ }^{27}$. However, in the low cardiorespiratory fitness group, the correspondence between HRVT measured using the RMSSD parameter and VT were similar, as reported by SALES et al. ${ }^{26}$ comparing HRVT through vagal-mediated indices and $\mathrm{VT}^{26}$.

Considering the differences between HRV indices through progressive exercise testing, the stabilization point using sympathetic or overall parameters (i.e., SDNN) occurs later than vagal-mediated indices ${ }^{5}$, ${ }^{25}$. Similarly, the $\mathrm{VO}_{2}$ at $\mathrm{HRVT}_{\text {RMSSD }}$ was lower than $V \mathrm{O}_{2}$ at $\mathrm{HRVT}_{\text {SDNN }}$ in both groups. However, no difference was found between VT and HRVT $\mathrm{SDNN}_{\text {N }}$ in the low or high cardiorespiratory fitness groups when expressed in $\mathrm{VO}_{2}$. This difference is probably related to the physiological significance of HRV indices which reflect the sympathetic and parasympathetic drives of cardiac autonomic control during exercise ${ }^{5,15}$. The RMSSD is considered as a parasympathetic parameter related to the autonomic control perspective, which leads to a faster decay rate during progressive exercise testing, while SDNN is influenced by both sympathetic and parasympathetic pathways ${ }^{1,5}$ and generally achieves asymptotic values after $\mathrm{VT}^{5}$. Similar values were found to our results when the percentage $\mathrm{VO}_{2}$ max at VT and HRVT $(-64 \%)$ were analyzed according to parasympathetic parameters ${ }^{22}$. The HRV by time domain has proven to be a valid way to examine

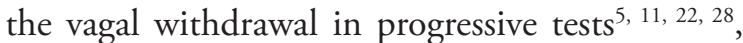
and high reliability was observed when ascertaining HRVT using the vagal parameter through visual inspection ${ }^{25}$, as performed in the present study.

While the $V O_{2}\left(\mathrm{ml} \bullet \mathrm{kg}^{-1} \bullet \mathrm{min}^{-1}\right)$ at VT, HRVT $_{\text {RMSSD }}$, and HRVT ${ }_{\text {SDNN }}$ was higher in the high cardiorespiratory fitness group, the percentage (\%) $\mathrm{VO}_{2}$ values were similar between the thresholds analyzed and the groups studied. Differences were found between groups for relative to body mass power output corresponding to VT and HRVT $_{\text {SDNN }}$, but no difference was observed for $\mathrm{HRVT}_{\text {RMSSD }}$. In addition, although HRVT ${ }_{\text {SDNN }}$ displayed better correlations in the comparison with VT than HRVT $_{\text {RMSSD }}$, the use of the vagally mediated RMSSD throughout the progressive test appears to discriminate cardiorespiratory fitness levels (FIGURE 1, panel A) considering percentages of workloads near to the VT or HRVT $(60-80 \%$ of $\mathrm{VO}_{2}$ max). Conversely, SDNN did not differ between groups (FIGURE 1, panel B) at any workload. These results confirm the assumption that vagal indices are higher in individuals with good cardiorespiratory fitness levels during progressive exercise testing ${ }^{15}$. This could be due to a physiological concept, the higher 'parasympathetic reserve' present in the high cardiorespiratory fitness group, as previously observed ${ }^{5,6,15}$. In this sense, the use of a vagal HRV parameter analyzed by RMSSD was sensitive to discriminate physical fitness for initial workloads comparing the low and high cardiorespiratory fitness groups. Thus, our results suggest, according to ES, the use of a vagal HRV parameter as a method able to discriminate by 
cardiac vagal modulation withdrawal throughout progressive exercise and its relationship with cardiorespiratory physical fitness, as highlighted by other studies ${ }^{14,15}$

A greater relationship between VT and HRVT $_{\text {RMSSD }}$ than the overall (SDNN) HRV parameter as assessed by $\mathrm{HRVT}_{\text {SDNN }}$ was expected, as observed previously ${ }^{5,11,22}$. However, the present study demonstrated a significant correlation only between the $\mathrm{VO}_{2}$ at $\mathrm{VT}$ and $\mathrm{HRVT}_{\text {SDNN }}(r=0.77)$ for the low cardiorespiratory fitness group and a weaker non-significant correlation $(r=0.39)$ for the high cardiorespiratory fitness group. The Pearson's correlation presented lower values between the

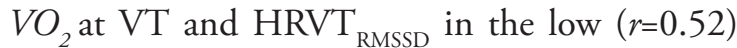
and high $(r=0.38)$ cardiorespiratory fitness groups. This finding confirms the hypothesis that cardiorespiratory fitness level is an important factor to consider in HRVT evaluation and its physiologic mechanisms linked to the VT or metabolic transition should be revised. Different results were found to relate the VT with HRVT, using vagal parameters, $(r=0.69-0.97)$ by CoTTIN Con $_{22}$ inelltrained subjects and $(r=0.89)$ by Karapetian ${ }^{11}$ in non-athletes. This disagreement could be due to the different methods applied to determine HRVT or the profile of the individuals studied.

A strict methodological criterion was applied to determine VT and HRVT in the present study to minimize potential errors by using the visual inspection technique. In this way, physical fitness appears to be the main factor regarding HRVT determination, considering the results of individuals with high cardiorespiratory fitness. It is not possible to state precisely what physiologically led to such a result, whether there is a mechanistic link between cardiac autonomic response and VT during the incremental test. A larger relationship was found between the $\mathrm{VO}_{2}$ at VT with a vagal HRV parameter (SD1, an equivalent parameter of RMSSD) by TuLPPO ${ }^{5}$ and overall HRV parameter (SDNN) by YАмАмото 29 at the moment that the $\mathrm{RR}$ interval variability decreases progressively toward stability $(-60 \%$ of Wmax). To summarize, although HRVT has been validated previously with both LT and VT1 1,22, 26, the present study demonstrated that only $\mathrm{HRVT}_{\text {SDNN }}$ was correlated with VT in the low cardiorespiratory fitness group, therefore caution is recommended, especially with individuals with high cardiorespiratory fitness levels.
The increase in heart rate during exercise can be partly explained by the vagal withdrawal. The HR at HRVTRMSSD preceded the HR at $\mathrm{VT}$ in the low cardiorespiratory fitness group, as observed by $\mathrm{SHIBATA}^{30}$. In spite of this, in the present study the HR at HRVT RMSSD $_{\text {was }}$ not different from that observed in the VT and HRVT $_{\text {SDNN }}$. This disagreement may indicate that the physiological mechanisms may be partly distinct and that methodological aspects of VT, LT, and HRVT determination could contribute to mismatched results. The main reason for this is the higher variability of HRV during exercise observed in the high cardiorespiratory fitness group (see FIGURE 1C and 1D as an example), influenced by larger vagal tone ${ }^{15}$. Additionally, one advantage of HRVT through visual inspection is related to the fact that VT evaluation is an expensive method and LT is an invasive procedure, whereas HRVT through visual inspection using an $\mathrm{HR}$ monitor with $\mathrm{RR}$ interval recording (every $2 \mathrm{~min}$ per stage), besides being a non-invasive method, is easily applicable and highly reproducible ${ }^{25}$.

The limitations in the present study must be pointed out. The determination of VT and HRVT by visual inspection is open to some subjectivity, despite presenting higher reliability than HRVT measured by mathematical models ${ }^{25}$. To reduce this subjectivity, methodological criteria are applied using two, or in some cases three evaluators, which can lower the practicality of this method. In addition, the split of only two groups, the small sample size, and recruitment of only males makes it difficult to generalize our results to different populations. In this way, additional research is warranted to confirm our hypothesis and findings with a larger population using clustering analysis.

In conclusion, the main finding of this study was that cardiorespiratory physical fitness may affect HRVT evaluation and that the HRVT $_{\text {SDNN }}$ was closer to VT in the low cardiorespiratory fitness group than the high cardiorespiratory fitness group. However, the use of vagal modulation assessed by HRV, for which we used RMSSD, is more appropriate than SDNN to observe possible differences regarding cardiorespiratory fitness during progressive exercise testing. Thus, the use of HRVT as a tool to assess metabolic transition should be used with caution. 


\section{Acknowledgments}

The authors would like to thank the subjects who participated in the study and to thank Ricardo Oliveira and Robin Camargo for their helpful comments during the final version of this manuscript.

\section{Conflict of interest}

No conflicts of interest are declared by the authors.

\section{References}

1. Heart rate variability: standards of measurement, physiological interpretation and clinical use. Task Force of the European Society of Cardiology and the North American Society of Pacing and Electrophysiology. Circulation. 1996;93(5):1043-65. 2. Sandercock GR, Brodie DA. The use of heart rate variability measures to assess autonomic control during exercise. Scandinavian J Med Sci Sports. 2006;16(5):302-13.

3. Akselrod S, Gordon D, Ubel FA, Shannon DC, Berger AC, Cohen RJ. Power spectrum analysis of heart rate fluctuation: a quantitative probe of beat-to-beat cardiovascular control. Science (New York, NY). 1981;213(4504):220-2.

4. Hautala AJ, Makikallio TH, Kiviniemi A, Laukkanen RT, Nissila S, Huikuri HV, et al. Cardiovascular autonomic function correlates with the response to aerobic training in healthy sedentary subjects. Am J Physiol Heart Circulatory Physiol. 2003;285(4):H1747-52.

5. Tulppo MP, Makikallio TH, Takala TE, Seppanen T, Huikuri HV. Quantitative beat-to-beat analysis of heart rate dynamics during exercise. Am J Physiol. 1996;271(1 Pt 2):H244-52.

6. Buchheit M, Gindre C. Cardiac parasympathetic regulation: respective associations with cardiorespiratory fitness and training load. Am J Physiol Heart Circulatory Physiol. 2006;291(1):H451-8.

7. De Freitas VH, Pereira LA, de Souza EA, Leicht AS, Bertollo M, Nakamura FY. Sensitivity of the Yo-Yo Intermittent Recovery Test and Cardiac Autonomic Responses to Training in Futsal Players. Intern J Sports Physiol Perform. 2015;10(5):553-8.

8. Soares-Caldeira LF, de Souza EA, de Freitas VH, de Moraes SM, Leicht AS, Nakamura FY. Effects of additional repeated sprint training during preseason on performance, heart rate variability, and stress symptoms in futsal players: a randomized controlled trial. J Strength Conditioning Res. 2014;28(10):2815-26.

9. Wasserman K, Whipp BJ, Koyl SN, Beaver WL. Anaerobic threshold and respiratory gas exchange during exercise. J Applied Physiol. 1973;35(2):236-43.

10. Caiozzo VJ, Davis JA, Ellis JF, Azus JL, Vandagriff R, Prietto CA, et al. A comparison of gas exchange indices used to detect the anaerobic threshold. J Applied Physiol: respiratory, environmental and exercise physiology. 1982;53(5):1184-9. 11. Karapetian GK, Engels HJ, Gretebeck RJ. Use of heart rate variability to estimate LT and VT. Intern J Sports Med. 2008;29(8):652-7.

12. Hagberg JM, Coyle EF, Carroll JE, Miller JM, Martin WH, Brooke MH. Exercise hyperventilation in patients with McArdle's disease. J Applied Physiology: respiratory, environmental and exercise physiology. 1982;52(4):991-4.

13. Gaesser GA, Poole DC. Lactate and ventilatory thresholds: disparity in time course of adaptations to training. J Applied Physiol. 1986;61(3):999-1004.

14. Hautala AJ, Kiviniemi AM, Tulppo MP. Individual responses to aerobic exercise: the role of the autonomic nervous system. Neuroscie Biobehavioral Rev. 2009;33(2):107-15.

15. Tulppo MP, Makikallio TH, Seppanen T, Laukkanen RT, Huikuri HV. Vagal modulation of heart rate during exercise: effects of age and physical fitness. Am J Physiol. 1998;274(2 Pt 2):H424-9.

16. Koch B, Schaper C, Ittermann T, Spielhagen T, Dorr M, Volzke H, et al. Reference values for cardiopulmonary exercise testing in healthy volunteers: the SHIP study. Eur Resp J. 2009;33(2):389-97.

17. Poole DC, Wilkerson DP, Jones AM. Validity of criteria for establishing maximal O2 uptake during ramp exercisentests. Eur J Applied Physiol. 2008;102(4):403-10.

18. Borg GA. Psychophysical bases of perceived exertion. Med Sci Sports Exercise. 1982;14(5):377-81.

19. Amann M, Subudhi AW, Walker J, Eisenman P, Shultz B, Foster C. An evaluation of the predictive validity and 
reliability of ventilatory threshold. Med Sci Sports Exercise. 2004;36(10):1716-22.

20. Hughson RL, Green HJ, Sharratt MT. Gas exchange, blood lactate, and plasma catecholamines during incremental exercise in hypoxia and normoxia. J Applied Physiol. 1995;79(4):1134-41.

21. Cohen J. Statistical power analysis for the behavioral sciences. 2nd ed1988.

22. Cottin F, Medigue C, Lopes P, Lepretre PM, Heubert R, Billat V. Ventilatory thresholds assessment from heart rate variability during an incremental exhaustive running test. Intern J Sports Med. 2007;28(4):287-94.

23. Anosov O, Patzak A, Kononovich Y, Persson PB. High-frequency oscillations of the heart rate during ramp load reflect the human anaerobic threshold. Eur J Applied Physiol. 2000;83(4 -5):388-94.

24. Mendia-Iztueta I, Monahan K, Kyrolainen H, Hynynen E. Assessment of Heart Rate Variability Thresholds from Incremental Treadmill Tests in Five Cross-Country Skiing Techniques. PloS one. 2016;11(1):e0145875.

25. Candido N, Okuno NM, da Silva CC, Machado FA, Nakamura FY. Reliability of the Heart Rate Variability Threshold using Visual Inspection and Dmax Methods. Intern J Sports Med. 2015;36(13):1076-80.

26. Sales MM, Campbell CS, Morais PK, Ernesto C, Soares-Caldeira LF, Russo P, et al. Noninvasive method to estimate anaerobic threshold in individuals with type 2 diabetes. Diabetol Metabolic Syndrome. 2011;3(1):1-8.

27. Tulppo MP, Hautala AJ, Makikallio TH, Laukkanen RT, Nissila S, Hughson RL, et al. Effects of aerobic training on heart rate dynamics in sedentary subjects. J Applied Physiol. 2003;95(1):364-72.

28. Vasconcellos F, Seabra A, Montenegro R, Cunha F, Bouskela E, Farinatti P. Can Heart Rate Variability be used to Estimate Gas Exchange Th reshold in Obese Adolescents? Intern J Sports Med. 2015;36(8):654-60.

29. Yamamoto Y, Hughson RL, Peterson JC. Autonomic control of heart rate during exercise studied by heart rate variability spectral analysis. J Applied Physiol. 1991;71(3):1136-42.

30. Shibata M, Moritani T, Miyawaki T, Hayashi T, Nakao K. Exercise prescription based upon cardiac vagal activity for middle-aged obese women. International journal of obesity and related metabolic disorders. J Intern Ass Study Obesity. 2002;26(10):1356-62.

\begin{tabular}{r|r} 
ADDRESS & \\
Lúcio Flávio Soares Caldeira & \\
Universidade Norte do Paraná & Submitted: 06/27/2017 \\
Departamento de Educação Física & Revised: 01/18/2018 \\
Centro de Ciências Biológicas e da Saúde & Accepted: 03/29/2018 \\
Av. Paris, 675 - Campus Universitário & \\
86041-120 - Londrina - PR - Brazil & \\
E-mail: luciocaldeira@yahoo.com.br & \\
&
\end{tabular}

